\title{
Multiple controls affect arsenite oxidase gene expression in Herminiimonas arsenicoxydans
}

\author{
Sandrine Koechler ${ }^{1 \dagger}$, Jessica Cleiss-Arnold ${ }^{1 \dagger}$, Caroline Proux ${ }^{2}$, Odile Sismeiro², Marie-Agnès Dillies ${ }^{2}$, \\ Florence Goulhen-Chollet ${ }^{1}$, Florence Hommais ${ }^{3}$, Didier Lièvremont ${ }^{1}$, Florence Arsène-Ploetze ${ }^{1}$, Jean-Yves Coppée ${ }^{2}$, \\ Philippe N Bertin ${ }^{1 *}$
}

\begin{abstract}
Background: Both the speciation and toxicity of arsenic are affected by bacterial transformations, i.e. oxidation, reduction or methylation. These transformations have a major impact on environmental contamination and more particularly on arsenic contamination of drinking water. Herminiimonas arsenicoxydans has been isolated from an arsenic- contaminated environment and has developed various mechanisms for coping with arsenic, including the oxidation of $\mathrm{As}(\mathrm{III})$ to $\mathrm{As}(\mathrm{V})$ as a detoxification mechanism.

Results: In the present study, a differential transcriptome analysis was used to identify genes, including arsenite oxidase encoding genes, involved in the response of $\mathrm{H}$. arsenicoxydans to As(III). To get insight into the molecular mechanisms of this enzyme activity, a Tn5 transposon mutagenesis was performed. Transposon insertions resulting in a lack of arsenite oxidase activity disrupted aoxR and aoxS genes, showing that the aox operon transcription is regulated by the AoxRS two-component system. Remarkably, transposon insertions were also identified in rpoN coding for the alternative N sigma factor $\left(\sigma^{54}\right)$ of RNA polymerase and in dnaJ coding for the Hsp70 co-chaperone. Western blotting with anti-AoxB antibodies and quantitative RT-PCR experiments allowed us to demonstrate that the $r p o N$ and dnaJ gene products are involved in the control of arsenite oxidase gene expression. Finally, the transcriptional start site of the aoxAB operon was determined using rapid amplification of CDNA ends (RACE) and a putative -12/-24 $\sigma^{54}$-dependent promoter motif was identified upstream of aoxAB coding sequences.
\end{abstract}

Conclusion: These results reveal the existence of novel molecular regulatory processes governing arsenite oxidase expression in $\mathrm{H}$. arsenicoxydans. These data are summarized in a model that functionally integrates arsenite oxidation in the adaptive response to As(III) in this microorganism.

\section{Background}

Arsenic is present in various environments, released from either anthropogenic or natural sources. This element is toxic for living organisms and known to be a human carcinogen [1]. Its toxicological effects depend, at least in part, on its oxidation state and its chemical forms, inorganic species being considered as more toxic [2]. The contamination of drinking water by the two inorganic forms, arsenite $\mathrm{As}(\mathrm{III})$ and arsenate $\mathrm{As}(\mathrm{V})$, has been reported in different parts of the world [3] and constitutes a major threat of public health. Microorganisms are known to take part in the transformation, i.e

\footnotetext{
*Correspondence: philippe.bertin@unistra.fr

+ Contributed equally

'UMR7156 Génétique Moléculaire, Génomique et Microbiologie, CNRS

Université de Strasbourg, 28 rue Goethe, 67000 Strasbourg, France
}

oxidation, reduction or methylation of the metalloid, having a deep impact on arsenic contamination in environment.

Several bacteria and prokaryotes have developed adaptation, resistance and colonization mechanisms, which allow them to live in hostile arsenic contaminated environments. $H$. arsenicoxydans is a Gram-negative $\beta$-proteobacterium isolated from an industrial activated sludge plant and exhibiting a remarkable set of arsenic resistance determinants [4]. The $H$. arsenicoxydans adaptive response to arsenic is organized in a complex and sophisticated network. In particular, differential proteome studies have recently demonstrated the synthesis of several proteins encoded by the three ars resistance operons, e.g. arsenate reductase $\mathrm{Ars} \mathrm{C}$, flavoprotein $\mathrm{ArsH}$ and regulator ArsR $[5,6]$ and the induction of oxidative
Ciomed Central

() 2010 Koechler et al; licensee BioMed Central Ltd. This is an Open Access article distributed under the terms of the Creative Commons Attribution License (http://creativecommons.org/licenses/by/2.0), which permits unrestricted use, distribution, and reproduction in any medium, provided the original work is properly cited. 
stress protein encoding genes, e.g. catalase (katA), superoxide dismutase $(\operatorname{sod} B)$ and alkyl hydroperoxide reductase $(a h p C)$ [7].

One of the most noticeable response to arsenic in $H$. arsenicoxydans is the ability of this bacterium to oxidize As(III) to As(V), a less toxic and less mobile form, via an arsenite oxidase activity. The two genes coding for this heterodimeric enzyme are organized in an operonic structure, and have been named $a \circ x A$ and $a \circ x B$ for the small and the large subunit, respectively $[6,8,9]$. Homologous genes have been since identified in various microorganisms [6,10-13]. In Agrobacterium tumefaciens, a complex transcriptional regulation has been recently suggested, involving As(III) sensing, two-component signal transduction by an AoxS sensor kinase and an AoxR regulator, and quorum sensing [14]. Nevertheless, the molecular mechanisms involved in the control of arsenite oxidase expression remain largely unknown.

To extend our view of arsenic response [7], in particular with respect to the arsenic oxidation process, we conducted a comprehensive transcriptomic, genetic and molecular analysis of $H$. arsenicoxydans following exposure to As(III). These approaches allowed us to identify major determinants involved in the control of arsenite oxidation.

\section{Results}

Gene expression profiling in response to arsenic

The response to As(III) was analyzed in exponentially growing cells using microarrays. The data from three biological replicates were combined after normalization and statistical analysis carried out using the $\mathrm{R}$ software and packages http://www.r-project.org. The set of genes was further refined to include only those genes that showed a valid $p$-value and whose expression was altered by a factor of 2 or more when compared to the level measured in the absence of arsenic.

This experiment led to the identification of 293 genes showing an arsenic-induced expression change ( $>2$ fold $\left.\left(\log _{2}=1\right)\right)$. Among these genes, $133(45 \%)$ were upregulated and the remaining part, i.e. 160 genes, was down-regulated. The relative changes in gene expression ranged from a 11-fold down-regulation ( $r p s N$ gene encoding a ribosomal protein) to a 126 -fold up-regulation (putative gene involved in phosphate transport). A list of those genes is shown in Additional file 1, Table $\mathrm{S} 1$. The corresponding HEAR gene numbers are available in the Arsenoscope relational database http://www. genoscope.cns.fr/agc/mage/arsenoscopevia the MaGe web interface [15].

The 293 genes differentially expressed were clustered according to the function of the corresponding encoded proteins. Among the 133 genes that were induced by at least a 2 -fold factor, about $11 \%$ are involved in metabolism, 26\% in transport and binding protein, $26 \%$ in cellular processes and $31 \%$ have no assigned function. The high percentage of genes with unknown function is in accordance with the proportion of unknown function proteins identified in the genome of $H$. arsenicoxydans $[6,7]$. In agreement with our previous results, genes involved in arsenic resistance, phosphate transport and flagellar biosynthesis were induced in the presence of As (III) (see Additional file 1, Table S1), further supporting the relationship between these physiological processes $[6,7]$. Interestingly, only one methyl-accepting chemotaxis protein (MCP) gene was induced in our microarray data, suggesting a role for this protein in the sensing of arsenic. This mechanism is currently under investigation. Genes encoding the putative nitroreductase AoxC and the cytochrome $\mathrm{C}_{552}$ precursor AoxD as well as the response regulator AoxRS were found to be induced by As(III) (see Additional file 1, Table S1). AoxR has been proposed to act as a positive regulator of the aox operon upon phosphorylation by AoxS in A. tumefaciens [14]. Our transcriptomic data suggest that the regulation machinery is, at least in part, similar in $H$. arsenicoxydans. Futhermore, genes coding for the arsenite oxidase AoxAB subunits were found to be among the most induced genes in the presence of As(III). However, even though these results extended our knowledge of the arsenic response in $H$. arsenicoxydans, they did not led to a better understanding of the molecular mechanisms involved in the control of arsenite oxidation. This prompted us to perform a transposon mutagenesis experiment.

\section{Identification of arsenite oxidase accessory genes by screening an Aox activity deficient mutant library}

To identify genes possibly involved in the control of arsenite oxidation in $H$. arsenicoxydans, a library of 10,000 kanamycin resistant mutants was constructed by transposon mutagenesis, as previously described [9]. These clones were tested by silver nitrate staining [16] for arsenate production on As(III)-supplemented CDM agar plates. As compared to the wild-type strain, whose arsenite oxidase activity was revealed by a brownish precipitate, 10 mutants with a lack of As(III) oxidase activity were obtained. These strains showed no precipitate (Figure 1A), as observed for the M1 and M2 strains used as negative controls. Indeed, these strains carry a mutation in $a \circ x A$ or $a \circ x B$ genes coding for the small and the large subunit of arsenite oxidase, respectively [9]. Genes disrupted by transposon insertions were identified in these 10 new mutants. As expected, four of the 10 mutants showed insertions in the $a o x A B$ operon (Figure 2A). More interestingly, six mutants carried a transposon insertion outside the $\operatorname{aox} A B$ operon. Two 

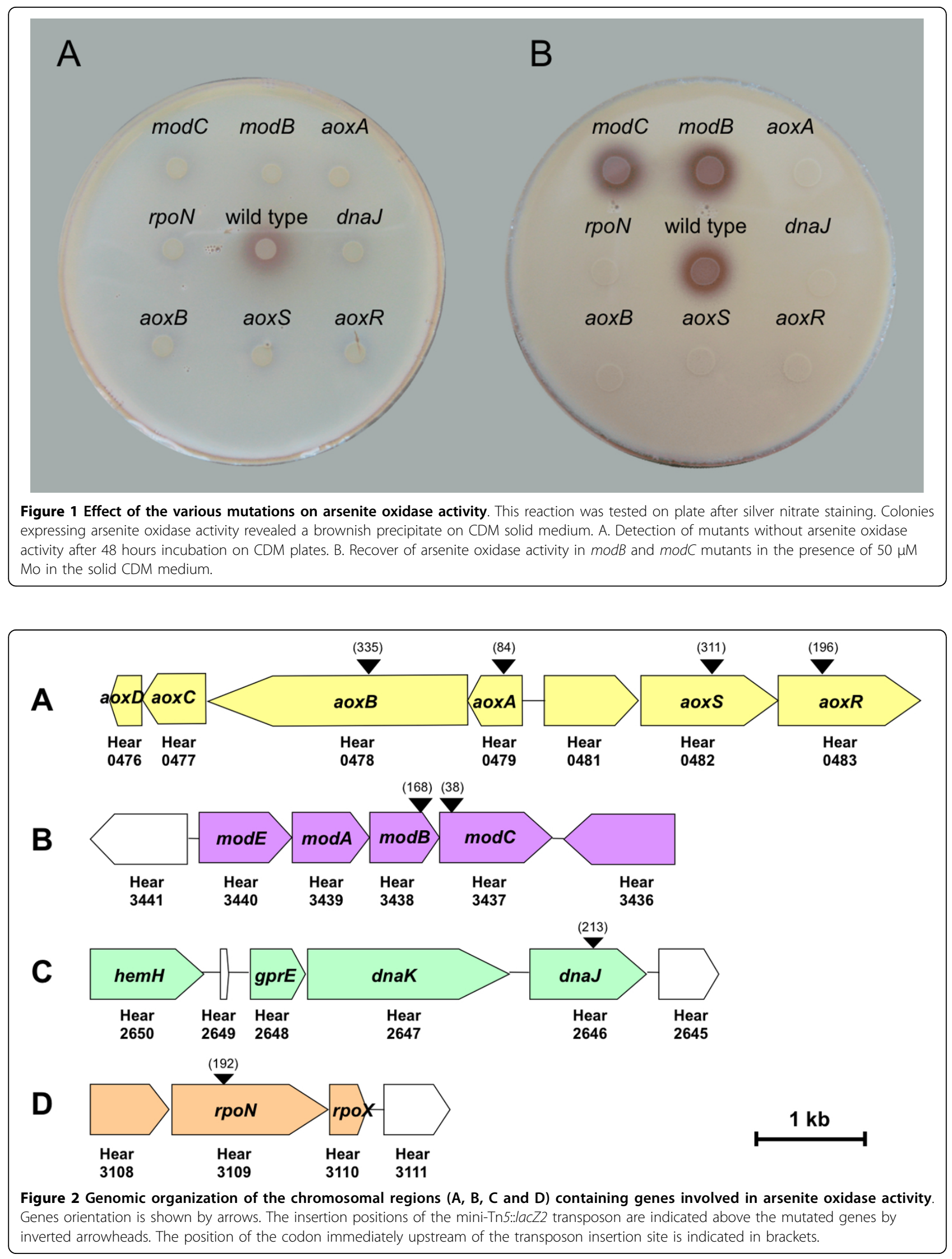
mutants were found to be affected in the aoxRS twocomponent signal transduction system (mutants $\mathrm{Ha} 482$ and Ha483, respectively) located upstream of the $a \circ x A B$ operon in $H$. arsenicoxydans [6] (Figure 2A). These results further support our transcriptomic data suggesting that these two genes play a role in arsenic response. Two transposon insertions were shown to disrupt genes of the modEABC operon coding for a molybdenum high-affinity transport system [17], i.e. $\bmod C$ encoding an ATP-binding cassette transport protein (mutant Ha3437) and $\bmod B$ encoding a molybdenum transport system permease (mutant Ha3438) (Figure 2B). Remarkably, transposon insertions were also located in $d n a J$ encoding a heat shock protein (Hsp40), (mutant Ha2646) (Figure 2C) and in rpoN encoding the alternative nitrogen sigma factor (sigma 54) of RNA polymerase (mutant Ha3109) (Figure 2D).

Two additional experiments were performed to complete the physiological characterization of these mutants with respect to arsenite oxidation. First, arsenic species were quantified by HPLC-ICP-AES on filtered culture supernatants. $H$. arsenicoxydans was grown in liquid medium supplemented with $1.33 \mathrm{mM}$ arsenite and showed $100 \%$ transformation of As(III) into As(V) after $48 \mathrm{~h}$, whereas M1 $(a \circ x A)$ and M2 $(a \circ x B)$ mutants used as controls were not able to transform As(III) into As (V). The same loss of arsenite oxidase activity was measured in Ha482 (aoxS), Ha483 (aoxR), Ha2646 (dnaJ) and $\mathrm{Ha} 3109(\mathrm{r} p o N)$ mutants. In contrast to the results obtained on agar plates, Ha3437 $(\bmod C)$ and Ha3438 $(\bmod B)$ strains showed $100 \%$ transformation of arsenite (Table 1, Figure 1A). Previous studies have demonstrated that the bioavailability of metals or trace elements considerably varies according to the type of matrix used for microbial growth [18]. We therefore assumed that Mo might be partly sequestred on CDM agar medium, resulting in a lack of arsenite oxidase

Table 1 Determination of arsenic speciation in $\boldsymbol{H}$. arsenicoxydans wild-type and mutant strains.

\begin{tabular}{llll}
\hline Strain & Mutated gene & \multicolumn{2}{l}{ Arsenic species identified $^{\text {a }}$} \\
\hline & & As(III) & As(V) \\
\hline ULPAs1 & $/$ & - & + \\
M1 & aoxA & + & - \\
M2 & aoxB & + & - \\
Ha482 & aoxS & + & - \\
Ha483 & aoxR & + & - \\
Ha2646 & dnaJ & + & - \\
Ha3109 & rpoN & + & - \\
Ha3437 & modC & - & + \\
Ha3438 & modB & - & + \\
\hline
\end{tabular}

${ }^{a}$ Determined by HPLC-ICP-AES after $48 \mathrm{~h}$ growth in CDM medium containing $100 \mathrm{mg}$.liter of arsenite.

b [9] activity on plate. To test this hypothesis, As(III) oxidase tests were performed on CDM agar plates supplemented with $50 \mu \mathrm{M}$ Mo. The addition of Mo to the solid medium restored As(III) oxidase activity in both $\mathrm{Ha} 3437$ $(\bmod C)$ and Ha3438 $(\bmod B)$ mutants while it had no effect on other mutant strains (Figure 1B).

Second, we have previously demonstrated that the polar flagellum-dependent motility of $H$. arsenicoxydans is increased in the presence of As(III), suggesting that arsenite oxidation may result in a gain of energy [6]. The motility of mutant strains was therefore tested on plates containing different concentrations of $\mathrm{As}(\mathrm{III})$, i. e. $0.66 \mathrm{mM}, 1.33 \mathrm{mM}$ and $2 \mathrm{mM}$. The diameter of the swarming rings was measured after $72 \mathrm{~h}$. As shown in Figure 3, the disruption of $\operatorname{aox} A$, $a \circ x B$, $a \circ x R$, $a \circ x S$ or rpoN genes abolished the improvement of swarming performances in the presence of As(III). Unlike those mutants, a disruption in $d n a J$ completely abolished the motility of $H$. arsenicoxydans in the presence or the absence of As(III). DnaJ is known to be essential for the expression of the $f l h D C$ flagellar master operon in Escherichia coli [19]. The lack of motility observed in the dnaJ mutant suggests the existence of a similar flhDC-dependent regulation of flagellar genes in $H$. arsenicoxydans. More importantly, the dnaJ mutant was affected in both the motility and arsenite oxidation, suggesting that these two mechanisms are co-regulated.

\section{Effect of AoxR, AoxS, RpoN and DnaJ on arsenite oxidase} synthesis

To get further insight into the involvement of AoxR, AoxS, RpoN and DnaJ in arsenite oxidase activity, Western immunoblotting experiments were performed using antibodies raised against AoxB. The abundance of this protein was evaluated from total protein extracts of $H$. arsenicoxydans wild-type and mutant strains grown in the presence or not of $\mathrm{As}(\mathrm{III})$. AoxB was detected as a single band corresponding to a molecular mass of 92 $\mathrm{kDa}$ in As(III)-challenged $H$. arsenicoxydans strain (Figure 4). This single band was not observed in the various mutant strains. Furthermore, arsenite oxidase activity on native gel was only detected in As(III)-challenged wild type total extract (data not shown). Taken together these results suggest that the lack of activity in the mutant strains is due to the absence of AoxB protein, which may result from an effect of AoxR, AoxS, RpoN and DnaJ on aoxAB expression.

\section{Effect of AoxR, AoxS, RpoN and DnaJ on the control of arsenite oxidase operon expression}

To determine the involvement of $a o x R, a o x S, d n a J$ and rpoN on $\operatorname{aox} A B$ transcription, we performed quantitative RT-PCR experiments. For each strain, changes in $a \circ x B$ transcript abundance were compared to two internal 


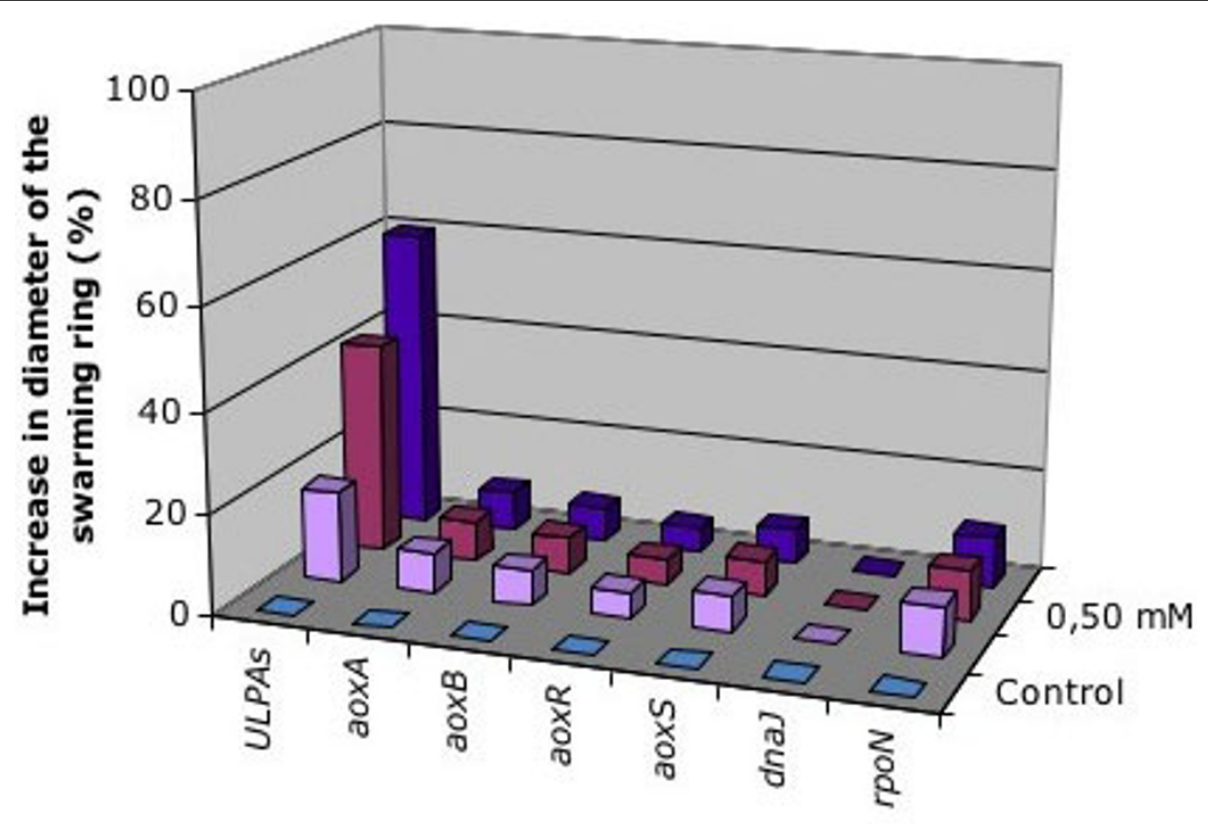

\section{$\square$ Control $\square 0,25 \mathrm{mM} \quad \square 0,50 \mathrm{mM} \quad \square 1 \mathrm{mM}$}

Figure 3 Effect of arsenite concentration on swarming properties in $\mathbf{H}$. arsenicoxydans wild-type and mutant strains. Motility assays were performed in the presence of an increased concentration of As(III). The level of motility of each strain was evaluated as the diameter of the swarming ring expressed in $\mathrm{mm}$. The results are the mean value of five independent experiments.

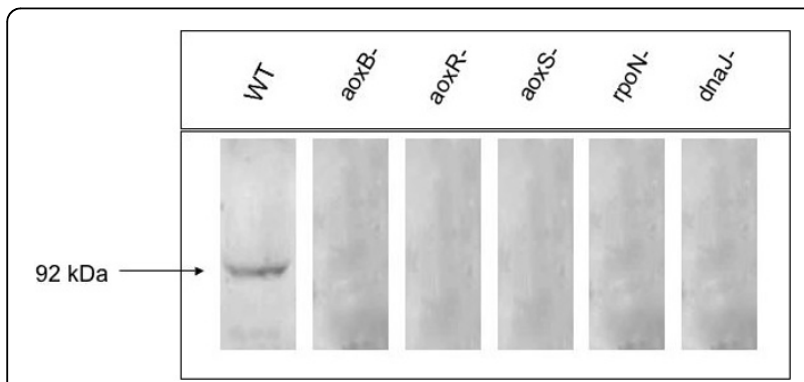

Figure 4 Immunodetection of AoxB protein in total protein extracts of $H$. arsenicoxydans wild-type and mutant strains

controls, i.e. the putative RNA methyltransferase gene and the peptide deformylase gene, in cultures challenged or not by As(III). The expression of $a \circ x B$ mRNA was increased by a 9.4 fold factor after As(III) exposure in the $H$. arsenicoxydans wild-type strain. In contrast, aoxB expression was not increased in Ha482 (aoxS), $\mathrm{Ha} 483$ (aoxR), Ha3109 (rpoN) and $\mathrm{Ha} 2646$ (dnaJ) mutant strains, suggesting that the corresponding proteins play a crucial role in aox $A B$ operon expression (Table 2).
Table 2 aoxB relative expression in $H$. arsenicoxydans wild-type and mutant strains.

\begin{tabular}{lll}
\hline Strain & $\begin{array}{l}\text { aoxB expression } \\
\text { ratio }+\mathbf{A s} \text { (III)/-As(III) }\end{array}$ & Standard error \\
\hline Wild type & 9.406 & 0.630 \\
Ha3109 (rpoN) & 0.250 & 0.060 \\
Ha483 (aoxR) & 0.111 & 0.024 \\
Ha482 (aoxS) & 0.200 & 0.029 \\
Ha2646 (dna) & 1.156 & 0.289 \\
\hline
\end{tabular}

Expression ratios of $a \circ x B$ in $H$. arsenicoxydans wild-type and mutant strains without $\mathrm{As}$ (III) versus an $\mathrm{As}$ (III) 8 hours induction $(1.33 \mathrm{mM})$, as measured by quantitative RT-PCR. Expression of each gene was normalized to the expression of the two housekeeping genes HEAR0118 and HEAR2922 coding for a peptide deformylase and a putative RNA methyltransferase, respectively. Standard errors were calculated from the data of two quantitative PCR replicates obtained from two independent biological replicates. Bold text indicates statistically significant induction.

\section{Molecular mechanisms of arsenite oxidase transcription}

The aox $R$ and aoxS genes encode a two-component system while rpoN encodes a sigma factor which recognizes a particular promoter with a specific $-12 /-24$ binding site. These three proteins may therefore play a role in the initiation of $a o x A B$ transcription. To get further insight into the molecular interactions between those regulators and the $a \circ x A B$ promoter, we mapped the 
transcriptional start site of this operon by the amplification of $\operatorname{aox} A B$ cDNA ends and 5'RACE. Messenger RNAs were extracted from induced (1.33 mM As(III)) and non induced $H$. arsenicoxydans wild-type strain cultures. A single transcriptional start site was identified from induced cells at -26 bp relative to the translation start codon, while no transcriptional start site was identified from non induced cells. In agreement with this, a TGGCACGCAGTTTGC putative $-12 /-24 \sigma^{54}$-dependent promoter motif was identified upstream of the aox $A B$ transcriptional start site (Figure 5). In addition, multiple alignment of $a o x A B$ promoter sequences present in databases revealed a similarity to promoters recognized by $\sigma^{54}$ in A. tumefaciens, Thiomonas sp., Rhizobium sp. NT-26, Achromobacter sp., Rhodoferax ferrireducens, Ochrobactrum tritici (Figure 5A). In contrast, no such $\sigma^{54}$-dependent promoter motif was found in several strains containing the $\operatorname{aox} A B$ operon but lacking the two-component transduction system aoxRS operon, such as Chloroflexus aurantiacus, Chlorobium limicola,
Thermus thermophilus, Burkholderia multivorans, Roseobacter litoralis, Pseudomonas sp.TS44, Chlorobium phaeobacteroides and Chloroflexus aggregans (Figure 5B).

Remarkably, a multiple alignment of amino acid sequences revealed that AoxR shares significant homology with a number of $\sigma^{54}$ RNA polymerase transcriptional activators, i.e. $35.96 \%$ identity with ZraR and $35.26 \%$ identity with AtoC from E. coli K12. AoxR contains three conserved domains shared by most Enhancer Binding Proteins (EBP), namely a $\mathrm{N}$-terminal response regulator receiver domain (amino acids 18-130), a central $\sigma^{54}$ interaction domain (amino acids 147-368) common to all $\sigma^{54}$ dependent EBPs (Pfam E-value 10 ${ }^{-116}$; http://www.sanger.ac.uk/cgi-bin/Pfam/getacc?PF00158) and a C-terminal DNA binding helix-turn-helix (HTF) domain (amino acids 421-463) enable to bind to specific upstream activation sequences [20]. AoxR shares similarities with several EBPs of $\sigma^{54}$ essential for the formation of an open complex formation during $\sigma^{54}$. dependent transcriptional initiation, in particular the $\sigma^{54}$

A

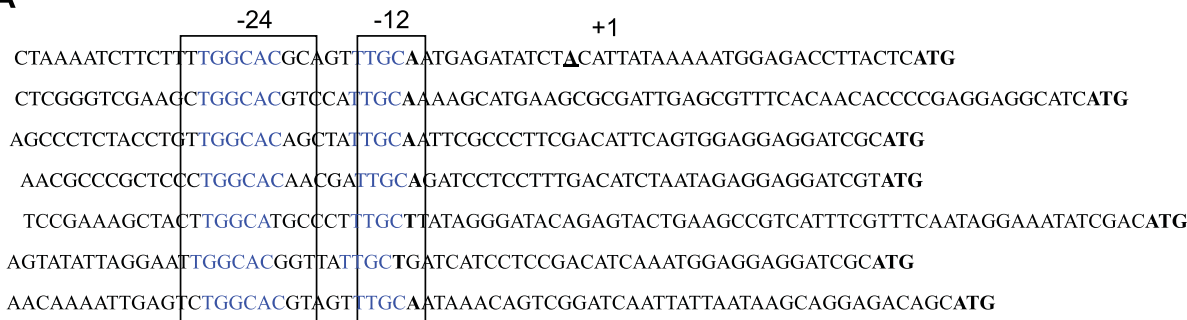

Herminiimonas arsenicoxydans

Thiomonas sp. 3As

Agrobacterium tumefaciens

Rhizobium sp. NT-26

Achromobacter sp. SY8

Ochrobactrum tritici

Rhodoferax ferrireducens $\mathrm{T118}$

\section{B}

TGACACGACCCGGCCAGGCCCCCCTTGACGAATGTCCCTACCGTTTGATAGTCTTCAAACGAAACAAAAGGGAGGTAAATATG TTCTCCTCTTTGAGATCGTTGACCTATACTAACAGTATCCAGCAGCTATCAGATGTCATACAATGCAGTGGAGGGAGGTCTATG ATCGGGTTGAGACCTCCCTGCGTTGCAGTTTTTTTCTCTGTCGCCAGTCATTCACAATTGTTATCAAATAAAGGAGGCATCCATG TTGTGTCCGGCTATCCGGGCATCTTGAAATTCCTCAAGGTTTGCCCTACACCAAAACCAACGATTCAATAAATATAGAAATATG ACAAGAAGTTGCGTCCGCCACCATTTCGATTGGAATCCAGTTCGTACATGCGATCAAGCAATCCGCCTATCAAATGTCGAAATG GGTCGTCACGACCTCGATCGGAATCCAATTCGGACCGCATTCAAACGACCCGTTCGTCCGATGCCGCCACGGAGGCGACCATC GTCCGCCACCATTTCGATTGGAATCCAGTTCGTACATGCGATCAAGCAATCCGCCTATCAAATGTCGAAATGGAGGAGACCATG CGACGATGACATCTATGCCGTGGGCATGTTCGGGCTGATTTACGGCCGTTTTGATAATCTGCAAGGCTGAGGAGAAACCCAATG GCATATATGAATTTTGTAATGAAAGCGTCATCGGTACGGATTTAGGTTGCGTCCCACCTCAACCCAATGCAGGAATCTACGATC TCTTTATAAATTCCACTTTTTCAGATTTTTTTTTGCCCAACATTGTTAACAATTGTTAGCACCAATAGCGAGGAGACCATACATG TTCTCTCTTGACAATTATCCCTAAAGATGATAATATGTATTCCACACAAGTATAAGTATCAATCAGGTTGGAGTACACGTCTATG
Thermus thermophilus $\mathrm{HB8}$ (plasmid) Chloroflexus aurantiacus J-10-fl Chlorobium limicola DSM 245 Burkholderia multivorans (Bmul5679)

Burkholderia multivorans (asoB I) Burkholderia multivorans (asoB II) Burkholderia multivorans (Bmul5703)

Roseobacter litoralis Och149 Pseudomonas sp. TS44

Chlorobium phaeobacteroides BS1 Chloroflexus aggregans DSM9485

Figure 5 Determination of aoxA transcription start site by $5^{\prime}$ RACE and identification of a $\sigma^{54}$ consensus motif. The transcription start site (TSS) of aoxA is in bold and indicated as +1 in the aoxA promoter sequence. The -12 and -24 boxes are highlighted and the consensus sequence is indicated in bold. The aoxA promoter was also aligned with the promoter sequences of $A$. tumefaciens, Thiomonas sp., Rhizobium sp. NT-26, Achromobacter sp., R. ferrireducens, O. tritici, C. aurantiacus, C. limicola, T. thermophilus, B. multivorans, R. litoralis, Pseudomonas sp.TS44, C. phaeobacteroides and C. aggregans. Two distincts sequences were shown A. DNA sequences with a $\sigma^{54}$-dependent promoter motif (indicated in boxes). B. DNA sequences without a $\sigma^{54}$-dependent promoter motif. Sequence informations of other genes were obtained from GenBank database and their localization on the chromosome or the plasmid is given by a nucleotide numbering. Their accession numbers are: $A$. tumefaciens (ABB51929.1), Thiomonas sp. (ABY19317.1), Rhizobium sp. NT-26 (AAR05655.1), Achromobacter sp. (ABP63659.1), R. ferrireducens (YP_524326.1), O. tritici (ACK38266.1), C. aurantiacus (YP_001634828.1), C. limicola (YP_001942455.1), T. thermophilus (YP_145367.1), B. multivorans (YP_001585660.1, YP_001941631.1, YP_001941634.1, YP_001585641.1), R. litoralis (ZP_02142508.1), Pseudomonas sp.TS44 (ACB05952.1), C. phaeobacteroides (YP_001960746.1) and C. aggregans (YP_002461760.1). 
activator sequence GAFTGA loop 1 which directly binds to $\sigma^{54}$ conserved region III (Figure 6) [21]. Taken together, these observations strongly suggest that AoxR interacts directly with $\mathrm{RpoN}$ to initiate the transcription of aox $A B$ operon in $H$. arsenicoxydans.

\section{Discussion}

Despite many works devoted to arsenic metabolism in microorganisms, little is known about the regulation of arsenite oxidase activity. In the present study, the combination of transcriptomic, genetic and molecular data provided a comprehensive view of the role of various proteins in the control of arsenite oxidation in $H$. arsenicoxydans (Figure 7). We showed that some proteins play an indirect role in this process, as their presence is not essential for AoxAB synthesis. In this respect, the AoxB large subunit contains a Mo site required in arsenite oxidase enzymatic activity [22].
Ha3437 $(\bmod C)$ and Ha3438 $(\bmod B)$ mutations were located in the molybdenum high-affinity transport system operon, which further support the key role of this element in enzyme activity. In addition, the recovery of As(III) oxidase activity in these two mutants in the presence of an excess molybdenum suggests that Mo may also be transported through an alternative uptake system in mod mutants, e.g. a low-affinity uptake system involving non specific permeases such as HEAR0069, HEAR0154, HEAR1749 or HEAR2391 or a sulfate transport system, as described in E. coli mod mutants [23].

More importantly, our results suggest that AoxR and RpoN constitute a transcriptional complex that play a major role in the initiation of $a \circ x A B$ operon transcription. Three mutants, i.e. Ha482 (aoxS), Ha483 (aoxR) and $\mathrm{Ha} 3109(r p o N)$, were affected in this process. The amino acids sequence analysis of $H$. arsenicoxydans AoxR and AoxS revealed the existence in these proteins

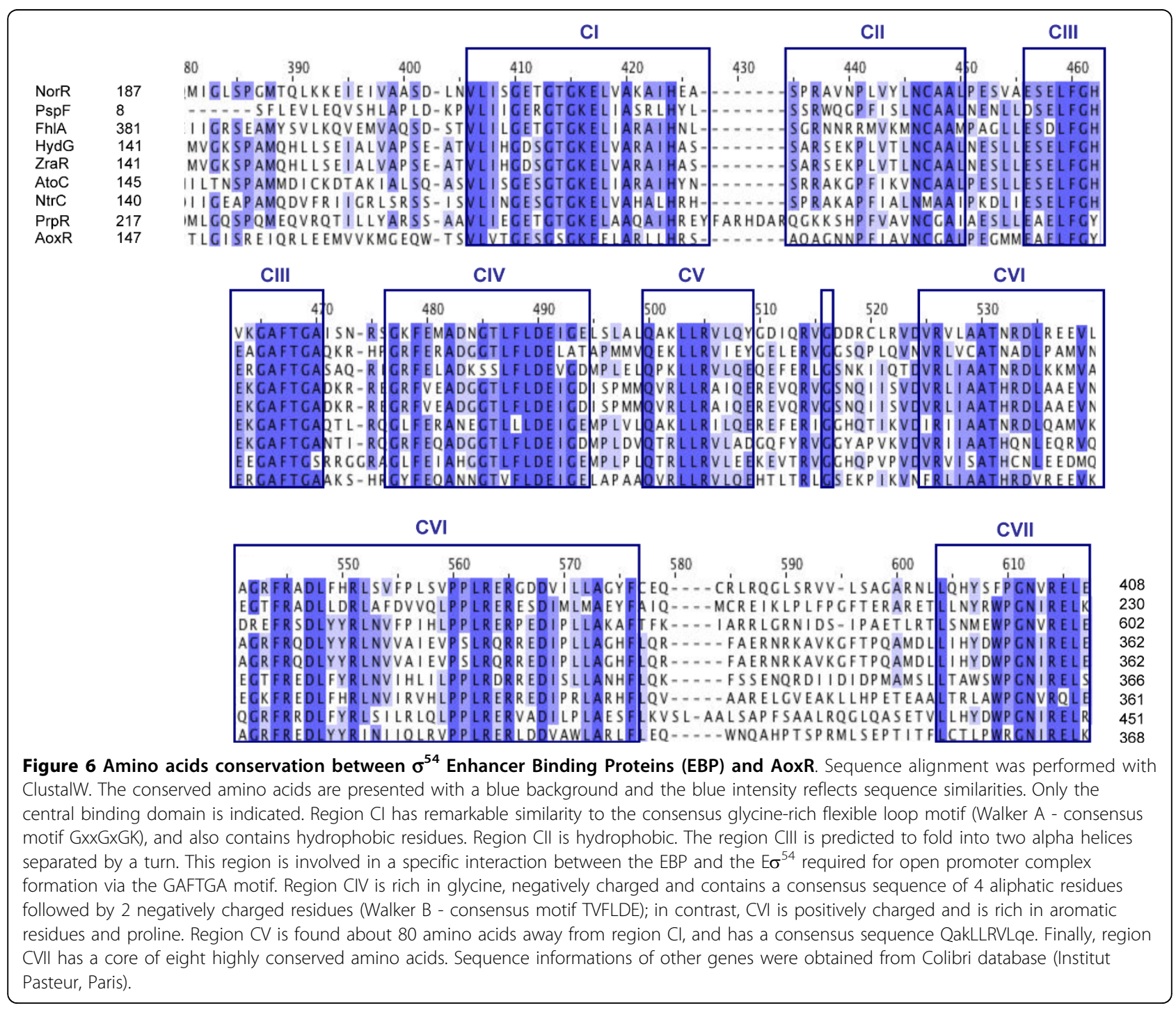




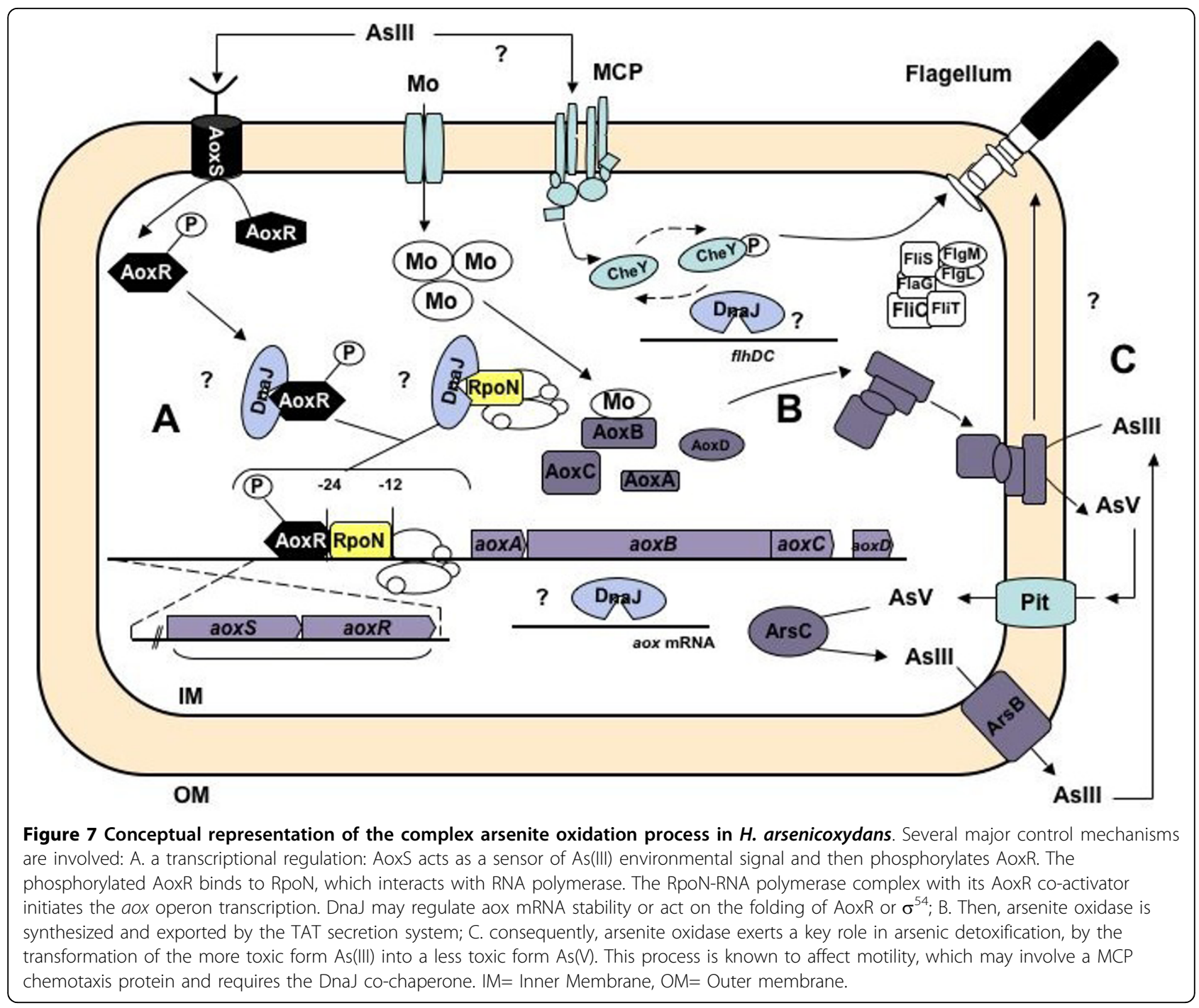

of structural features common to partners of two-component signal transduction systems, which are composed of a sensor kinase and a response regulator [24]. Moreover, the comparison of AoxS and AoxR protein sequences with those of $A$. tumefaciens revealed similarities. Indeed, the AoxS protein sequence contains short blocks of conserved motifs that are consistent with a role of sensor histidine kinase, e.g. the " $\mathrm{H}$ " (amino acids 279 to 287: LAHEVNNPL), the "G2" (amino acids 435 to 441: GRIGLGL) and the "N" (amino acids 380 to 391: VRQIVLNLVLNA) domains. In addition, four highly invariant residues playing a central role in phosphorylation correspond to Asp9, Asp10, Asp57 and Lys107 in the $H$. arsenicoxydans AoxR protein. The three asparagine residues are known to interact together to form an "acid pocket", characteristic of orthodox receivers, into which the side chain of Lys109 protrudes [24]. Finally, a transmembrane region and a 17 amino acids residue cluster possibly exposed to the periplasm are present in
AoxS and could serve as a signal receptor in the presence of As(III) in the medium. The detection of As(III) would then lead to AoxS autophosphorylation at a histidine residue via ATP hydrolysis and phosphotransfer to an aspartate residue in the response regulator AoxR, as recently proposed in A. tumefaciens [14].

Remarkably, our results demonstrated for the first time that the alternative $\mathrm{N}$ sigma factor $\left(\sigma^{54}\right)$ is essential for the initiation of arsenite oxidase transcription. Indeed, a mutation in the corresponding gene led to a complete loss of As(III) oxidation and $a \circ \times B$ transcription in Ha3109 (rpoN). $\sigma^{54}$ is one of the alternative sigma subunits of RNA polymerase responsible for specific binding to DNA. The core RNA polymerase complexed with $\sigma^{54}$ is usually associated with nitrogen assimilation and fixation, but is also known to play a role in various physiological processes, e.g. flagellar synthesis, carbon source utilization or bacterial virulence [25]. To date, only one report has shown that $\sigma^{54}$ 
participates in the transcription of genes possibly involved in metal tolerance, i.e. the $z \mathrm{raR} / \mathrm{S}$ genes that code for a zinc and lead responsive two-component regulatory system in $E$. coli [26]. RNA polymerase together with $\sigma^{54}$ binds to a specific promoter site, with the consensus DNA sequence YTGGCACGNNNN TTGCWNNw [27], forming a transcriptionally inactive closed complex. Such a characteristic -12/-24 $\sigma^{54}$-dependent promoter motif, i.e. TGGCACGCAGTTTGC, was identified $26 \mathrm{pb}$ upstream of the transcriptional initiation codon of $a \circ x A B$ with respect to the +1 transcriptional start site (Figure 5 ), which confirmed the need for RpoN in the initiation of $a \circ x A B$ transcription. Changes in the conformation of $\sigma^{54}$-RNA polymerase are nucleotide dependent. Indeed, the DNA melting step absolutely requires the interaction with a transcriptional activator protein. Most of these $\sigma^{54}$-dependent activators share three domains found in AoxR, i.e. a Cterminal DNA binding domain that binds to upstream activation sequences, a conserved central domain belonging to the AAA+ (ATPases associated with various cellular activities) protein family to proceed with initiation of transcription and a $\mathrm{N}$-terminal receiver domain that regulates its own AAA+ domain [20,28,29]. A multiple alignment of the central domain revealed a conservation of a common architecture between AoxR and $\sigma^{54}$ EBPs. Indeed, seven highly conserved sequence motifs corresponding to a $\sigma^{54}$ interaction domain of AoxR further support the direct interaction of AoxR with RpoN to stimulate the transcription of $a o x A B$ operon in $H$. arsenicoxydans (Figure 6). This central $\sigma^{54}$ interaction domain has been already used to identify new $\sigma^{54}$ EBPs [30-37]. It contains the highly conserved signature sequence GAFTGA loop (conserved region CIII, Figure 6) proved to be required for direct interaction with $\sigma^{54}[21,38,39]$, the Walker A (conserved region I, Figure 6) and B (conserved region CIV, Figure 6) ATPase motifs necessary for the open complex formation.

Both multiple sequences alignment and genetic environment analysis of aox promoters suggest the existence of a wide diversity in the transcriptional control of the aox operon. Indeed, as in $H$. arsenicoxydans, a $\sigma^{54}$ dependent promoter signature was identified in bacteria possessing a two-component transduction system AoxRS operon downstream of the aox $A B$ operon, e.g. $A$. tumefaciens and $O$. tritici (Figure 5A). In contrast, no $\sigma^{54}$-dependent promoter motif and no aox homologous gene were found in other bacteria, e.g. C. aurantiacus or C. aggregans (Figure 5B). These observations suggest that the transcription of the aox operon in these bacteria may involve other regulatory proteins and that AoxR may represent a specific co-activator of RpoN in the initiation of the aox operon transcription. Finally, our results provide evidence that the DnaJ co-chaperone is required for $\mathrm{As}$ (III) oxidation. DnaJ is part of the DnaK-DnaJ-GrpE Hsp70 machinery. Hsp70 chaperones represent one of the most potent defence cellular mechanism against environmental insults as DnaKDnaJ-GrpE are known to assist protein folding [40,41] or to be involved in mRNA stability [42]. In the present study we showed that there is no induction of $a \circ x A B$ transcription in the $d n a J$ mutant, resulting in a loss of AoxAB synthesis.

Several possible mechanisms involving DnaJ in the regulation of arsenite oxidase can be hypothesized. DnaJ may be required for the proper folding or activity of the AoxR regulator. Such a function has been demonstrated for the positive regulator CRP in a dnaJ deletion mutant in $E$. coli [43]. Similarly, a post-transcriptional regulation of the arsenite oxidase itself can not be excluded. Moreover, a Tat (Twin-Arginine Translocation) signal has been detected in the AoxA sequence of $H$. arsenicoxydans [6]. Proteins secreted to the periplasm via a Tat protein export pathway are known to require a folding by Hsp70 chaperones before their secretion. DnaJ could be one of these chaperones [44,45]. Another possible target of DnaJ may be the RpoN sigma factor, as this chaperone has been demonstrated to play a role in the regulation of $\sigma^{\mathrm{S}}$ in various species [46]. Alternatively, several mechanisms are known to be involved in the stability of messenger RNA. For example, in E. coli, a long 5' untranslated region (UTR) has been observed upstream of the transcriptional start site of the flhDC flagellum master operon. This region plays a crucial role in the stability of the mRNA controlled by CsrA [19]. In the present report, the $a \circ x A B$ transcriptional start site was located 26 bp upstream of the translational start codon, providing evidence that such a long 5'UTR does not exist upstream of the aox operon. However, this does not rule out a role of DnaJ on the stability of aox $A B$ mRNA, as HSP proteins are known to function as mRNA stabilizers and to protect them against nuclease degradation [42]. Further experiments will therefore be required to fully elucidate the molecular mechanisms of arsenite oxidase regulation in $H$. arsenicoxydans.

\section{Conclusion}

Taken together, our observations provide evidence that multiple proteins play a role in the control of arsenite oxidation in $H$. arsenicoxydans. The following regulatory model is proposed: AoxS responds to the presence of As(III) in the environment and autophosphorylates. The phosphate is then transferred to AoxR, which acts as a positive regulator of the aox operon and activates the initiation of the transcription in association with RpoN. In addition, DnaJ acts on the expression or the stability of both arsenite oxidation and motility genes, 
demonstrating that these two functions are strongly linked. Our results include the role of RpoN and DnaJ in arsenite oxidase synthesis, which provide further insight into the molecular mechanisms used by $H$. arsenicoxydans to cope with the most toxic form of arsenic in its environment.

\section{Methods}

\section{Bacterial strains and growth media}

Bacterial strains used in this study are listed in Table 3. $H$. arsenicoxydans ULPAs1 was grown in a chemically defined medium (CDM), supplemented by $2 \%$ agar when required [4]. Escherichia coli S17-1 strain [47] was cultivated in LB medium (MP Biochemicals). Matings were performed on CDM to which $10 \%$ (wt/vol) LB medium was added, as previously described [9]. Tryptone swarm plates containing CDM supplemented with 1\% Bacto-Tryptone and 0.25\% agar were used to assess bacterial motility.

\section{Transposon mutagenesis}

The mini-Tn5::lacZ2 transposon [47] was delivered by mobilization of the suicide vector $\mathrm{pUT} / \mathrm{mini}-\mathrm{Tn} 5$ ::lacZ2 from $E$. coli S17-1 ( $\lambda$-pyr) to H. arsenicoxydans. Conjugation was performed and transformants were selected as previously described [9].

\section{Selection of arsenite oxidase mutants}

Mutants were screened for arsenite oxidase activity as previously described [9]. Agar plates were flooded with a $0.1 \mathrm{M} \mathrm{AgNO}_{3}$ solution to visualize arsenite oxidation [16]. Mutants affected in molybdenum metabolism were also tested on CDM agar plates supplemented with 50 $\mu \mathrm{M} \mathrm{Na}{ }_{2} \mathrm{MoO}_{4}, 2 \mathrm{H}_{2} \mathrm{O}$ and $1.33 \mathrm{mM} \mathrm{As}(\mathrm{III})$.

\section{DNA manipulation and insertion mapping}

DNA manipulations were carried out according to standard protocols, as described by Sambrook et al. [48].

Table 3 Bacterial strains used in this study.

\begin{tabular}{lll}
\hline Name & Characteristics & Reference \\
\hline $\begin{array}{l}\text { Escherichia coli } \\
\text { S17-1 (-pyr) }\end{array}$ & pUT/miniTn5::lacZ2 & De Lorenzo et al., 1990 \\
Herminiimonas & & \\
arsenicoxydans & & \\
ULPAs1 & Wild type & Weeger et al., 1999 \\
M1 & aoxA::Tn5lacZ2 & Muller et al., 2003 \\
M2 & aoxB::Tn5lacZ2 & Muller et al., 2003 \\
Ha482 & aoxS::Tn5lacZ2 & This work \\
Ha483 & aoxR::Tn5lacZ2 & This work \\
Ha3437 & modC::Tn5lacZ2 & This work \\
Ha3438 & modB::Tn5lacZ2 & This work \\
Ha2646 & dnaJ::Tn5lacZ2 & This work \\
Ha3109 & rpoN::Tn5lacZ2 & This work \\
\hline
\end{tabular}

Total DNA was isolated from mutant strains with the Wizard Genomic DNA purification kit (Promega). Transposon insertion sites were mapped as previously described [9]. Briefly, total DNA was digested and selfligated. This ligation mixture was used as a template for PCR amplification using mini-transposon specific primers. The PCR products obtained were purified with a PCR purification kit (Qiagen) and sequenced on an Applied Biosystems ABI prism 3130×l capillary sequencer. The resulting sequences were compared to the $H$. arsenicoxydans genome sequence [6] to identify disrupted CDS. Finally, insertion sites and transposon orientations were precisely mapped by sequencing PCR products obtained with two primers hybridizing upstream and downstream, respectively, of the insertion site of each disrupted gene (see Additional file 2, Table S2).

\section{Arsenic speciation determination}

$H$. arsenicoxydans wild type and mutants were grown for 48 hours in CDM medium supplemented with 1.33 $\mathrm{mM}$ As(III). Culture supernatants were filtered through sterile $0,22 \mu \mathrm{m}$ pore size filters (VWR). Arsenic species were separated by high-performance liquid chromatography (HPLC) and quantified by inductively coupled plasma-atomic emission spectrometry (ICP-AES), as previously described [9].

\section{RNA extraction}

Strains were grown at $25^{\circ} \mathrm{C}$ for $24 \mathrm{~h}(\mathrm{OD}=0,15)$ and cultures were induced by addition of $0.66 \mathrm{mM}$ or 1.33 $\mathrm{mM}$ As(III) for 8 hours before extraction. Samples were harvested and stored at $-80^{\circ} \mathrm{C}$. RNA was extracted as previously described [7]. After extraction procedure, RNA integrity was checked by electrophoregram analysis on a BioAnalyser (Agilent) and total RNA concentration was determined spectrophotometrically with a Nanodrop.

\section{Microarrays and data analysis}

Microarrays containing 60-mer oligonucleotides for all predicted $H$. arsenicoxydans genes http://www.genoscope.cns.fr/agc/mage/arsenoscope were used, as previously described [7]. Briefly, total RNA $(5 \mu \mathrm{g})$ was reverse transcribed and indirectly labelled according to manufacturer's instructions with some modifications [7]. The quality and concentration determination as well as hybridization and scanning were performed as previously described [7] Three distinct biological RNA samples were prepared from in each growth condition (with and without As(III) induction) and labelled either by $\mathrm{Cy} 3$ or Cy5 in a dye-swap design. Microarray data were deposited in ArrayExpress (accession E-MEXP-2199 and A-MEXP-1594). Data normalization and statistical 
analysis were performed as previously described [7]. Briefly, data were acquired and analyzed by Genepix Pro 6.0 (Axon Instrument). The experiment design included three biological replicates. For each of them, induced and non-induced cells were compared in dye swap experiments. The resulting arrays were analyzed using the R software http://www.r-project.org. A slide by slide Loess normalization was performed using the limma package [49]. Valid $\log 2$ expression ratios from replicated spots were averaged on each array so as to get statistically independent ratios for each oligonucleotide included in the array design. For the same reason, dye swap arrays were also averaged. Oligos that had no valid expression ratios on the ten arrays were excluded from the data set for further analysis, which was carried out using the varmixt package and the VM option [50]. The resulting raw $p$-values were adjusted according to a Benjamini and Yekutieli procedure [51]. Genes showing a valid $p$-value and a more than two-fold decreased or increased expression were considered as differentially expressed between the two conditions and were retained for further study.

\section{Quantitative real time PCR}

Quantitative PCR experiments were performed with RNA prepared as described for microarrays. RNA aliquots were purified with the RNeasy Plus mini kit (Qiagen) to ensure the elimination of genomic DNA. Total RNA concentration was determined spectrophotometrically using a Nanodrop and RNA integrity was electrophoretically verified. Total RNA $(1,9 \mu \mathrm{g})$ was reverse transcribed with SuperScript III first-strand synthesis system for RT-PCR (Invitrogen) using random hexamers. Real time quantitative PCR was carried out with a MyiQ single-color Real-time PCR detection system. The reaction mixture contained 12,5 $\mu$ l of MESA Blue qPCR MasterMix Plus for SYBR Assay with fluorescein (Eurogentec), $5 \mu \mathrm{l}$ of cDNA and $300 \mathrm{nM}$ of each primer in a total volume of $25 \mu \mathrm{l}$. Thermocycling conditions were as follow: $5 \mathrm{~min}$ at $95^{\circ} \mathrm{C}$ and 40 cycles of $15 \mathrm{~s}$ at $95^{\circ} \mathrm{C}$, $15 \mathrm{~s}$ at $61^{\circ} \mathrm{C}$ and $1 \mathrm{~min}$ at $72^{\circ} \mathrm{C}$. The PCR efficiency of the genes of interest and internal control genes were optimized to be similar enough by adjusting the primer concentrations to $300 \mathrm{nM}$ each (data not shown). For each quantitative PCR run, non-template controls were performed to identify false positives and negative controls without reverse transcriptase were performed for each cDNA synthesis reaction and verified in real time PCR to determine the presence of contaminating genomic DNA. Two biological replicates (independent cultures) and two quantitative PCR replicates were performed for each experience. Amplification products were designed to be less than $175 \mathrm{bp}$ in size. The pairs of primers used are listed in Additional file 2, Table S2.
Two housekeeping genes, i.e. HEAR2922 coding for a putative RNA methyltransferase and HEAR0118 coding for a peptide deformylase, were used as standards to obtain normalized aoxB (HEAR0478) gene ratio [52] in the As(III) induced sample compared to the noninduced sample. These two housekeeping genes showed a stable expression between the two analyzed conditions (without As(III) and after an 8 hours As(III) exposure) when observing the microarrays data. The data were analyzed with the Relative Expression Software Tool [53]. Statistical significance was defined as a $p$-value of $\leq 0.05$.

\section{5'RACE experiment}

The transcriptional start site of $a o x A B$ operon was determined using the 5 'RACE system for rapid amplification of cDNA ends (Invitrogen). Total RNA was obtained as described before. Reverse transcriptase reactions were performed using $5 \mu \mathrm{g}$ total RNA and a gene specific reverse primer (5'-CATGGGCACTTGAATGTCTTG-3'). Reactions were heated at $70^{\circ} \mathrm{C}$ for $10 \mathrm{~min}$ and immediately prewarmed at $50^{\circ} \mathrm{C}$ before addition of Super-Script II reverse transcriptase. Reverse transcription was conducted at $50^{\circ} \mathrm{C}$ for $50 \mathrm{~min}$ and stopped at $70^{\circ} \mathrm{C}$ for $15 \mathrm{~min}$. Purification and tailing of cDNA were performed according to manufacturer's instructions. The resulting CDNA was amplified by PCR using the provided Abridged Anchor Primer and a gene specific primer (5'-ATGCTGTGCGCGACGATATCG$\left.3^{\prime}\right)$ located upstream of the original cDNA primer.

\section{Preparation of protein extracts, SDS-PAGE and PAGE separation}

Western immunoblotting were performed from late exponential phase wild-type and mutant strains grown in 1 liter CDM (with and without the presence of 100 $\mathrm{mg} /$ liter As(III)). The cultures were harvested by centrifugation for $10 \mathrm{~min}$ at $9,000 \times g$. Cell pellets were resuspended in distilled water and sonicated at $100 \mathrm{~A}$ (15 times 1 min with 1 min interval on ice, 80\% duty cycle). Cell debris were removed by centrifugation (15 min at $13,000 \times g$ ). The supernatant was collected (total extract) and stored at $-20^{\circ} \mathrm{C}$. The protein concentration of each sample was measured with a Bio-Rad protein assay kit. First, fifty micrograms of each protein extract was loaded onto an $11 \%$ polyacrylamide-SDS gel. Second, fifty micrograms of each protein extract were loaded onto a polyacrylamide gel (native gel). The assay of arsenite oxidase activity followed the transfer of reducing equivalents from arsenite to 2,4-dichlorophenolindophenol (DCIP) as described by Anderson et al. [54]. Briefly, the reduction of DCIP $(60 \mu \mathrm{M})$ was monitored in the presence of $200 \mu \mathrm{M}$ sodium arsenite in 50 $\mu \mathrm{M}$ MES, $\mathrm{pH} 6.0$, at $25^{\circ} \mathrm{C}$. 


\section{Preparation of antibodies and Western blot analysis}

Monoclonal antibodies raised against an AoxB peptide were obtained from Proteogenix. Briefly, a hexadecapeptide with the SKNRDRVALPPVNAQK sequence was synthesized. This peptide corresponds to the $\mathrm{N}$-terminal 16 amino acids of the arsenite oxidase large subunit of $H$. arsenicoxydans. The peptide was then coupled to keyhole limpet haemocyanin (KLH). Two rabbits were injected at multiple subcutaneaous sites with peptide$\mathrm{KLH}$ at 14 days intervals. Animals were prebled at day 0 , bled at day 49 (from an ear vein) and totally bled at day 90. Antibodies were partially purified on an affinity column substituted with the peptide.

After SDS-PAGE electrophoresis, the proteins were electrotransfered to a nitrocellulose membrane (Schleicher and Schuell, BA-85) using a Trans-Blot system (Bio-Rad) at $100 \mathrm{~V}, 4^{\circ} \mathrm{C}$ for $1 \mathrm{~h}$. The membranes were washed twice in Tris buffered saline (TBS: $10 \mathrm{mM}$ Tris$\mathrm{HCl} \mathrm{pH} 7.5,150 \mathrm{mM} \mathrm{NaCl}$ ) and blocked in TBS with $0,3 \%$ bovine serum albumin (BSA). The membrane was then washed three times in Tris-buffered saline with TritonX100 and Tween 20 (TBS-T: $20 \mathrm{mM}$ TrisHCl pH7.5, $500 \mathrm{mM} \mathrm{NaCl}, 0,2 \%$ Triton X-100, 0,05\% Tween20), and incubated for $1 \mathrm{~h}$ with the AoxB antisera (1:800 dilution) in TBS-T with 0,3\% BSA. Excess antibodies were removed by repeated washing with TBS-T. After 30 min incubation in TBS-T containing the secondary antibody (1:800 dilution of goat IgG against rabbit IgG, Sigma) conjugated with alkaline phosphatase, the membrane was washed twice with TBS-T and revealed by NBT/BCIP color reagent using standard procedures.

Additional file 1: Supplemental table S1. Selected genes differentially expressed after 8 hours arsenite stress.

Click here for file

[http://www.biomedcentral.com/content/supplementary/1471-2180-1053-S1.PDF ]

Additional file 2: Supplemental table S2. Oligonucleotides used in the study. A. Identification of transposon insertion sites in $\mathrm{H}$. arsenicoxydans mutants. B. Quantitative RT-PCR.

Click here for file

[http://www.biomedcentral.com/content/supplementary/1471-2180-1053-S2.PDF ]

\section{Acknowledgements}

JCA was supported by a grant from the French Ministry of Education and Research. Financial support came from the Centre National de la Recherche Scientifique, the Agence Nationale de la Recherche (ANR 07-BLAN-0118 project) and the Universite de Strasbourg. This work was done in the frame of the Groupement de Recherche (GDR2909-CNRS): « Métabolisme de I'Arsenic chez les Micro-organismes».

\section{Author details}

'UMR7156 Génétique Moléculaire, Génomique et Microbiologie, CNRS Université de Strasbourg, 28 rue Goethe, 67000 Strasbourg, France. ${ }^{2}$ Plateforme technologique Puces à ADN, Institut Pasteur, 28 rue du Dr. Roux,
75724 Paris cedex 15, France. ${ }^{3}$ UMR5240 Microbiologie, Adaptation et Pathogénie, CNRS Université Lyon 1, Bâtiment André Lwoff, 10 rue Dubois, 69622 Villeurbanne cedex France.

\section{Authors' contributions}

SK and JCA wrote the manuscript and performed the genetic experiments. SK carried out the quantitative PCR and 5' RACE experiments. JCA performed the Western immunoblotting analysis. CP, OS, MAD and JYC conceived and performed the transcriptomic experiments and the data analyses. DL performed the chemical experiments. FGC, FH, FAP and PB helped to analyze the data and critically revised the manuscript. PB coordinated and conceived the study. All authors read and approved the final manuscript.

Received: 26 October 2009

Accepted: 18 February 2010 Published: 18 February 2010

\section{References}

1. Mead MN: Arsenic: In search of an antidote to a global poison. Environ Health Perspect 2005, 113:A378-A386.

2. Rosen BP: Biochemistry of arsenic detoxification. FEBS Lett 2002, 529:86-92.

3. Smith $\mathrm{AH}$, Lingas EO, Rahman M: Contamination of drinking-water by arsenic in Bangladesh: A public health emergency. Bull World Health Organ 2000, 78:1093-1103.

4. Muller $D$, Simeonova DD, Riegel $P$, Mangenot $S$, Koechler $S$, Lièvremont $D$, Bertin PN, Lett MC: Herminiimonas arsenicoxydans sp. nov., a metalloresistant bacterium. Int J Syst Evol Microbiol 2006, 56:1765-1769.

5. Carapito C, Muller D, Turlin E, Koechler S, Danchin A, Van Dorsselaer A, Leize-Wagner E, Bertin PN, Lett MC: Identification of genes and proteins involved in the pleiotropic response to arsenic stress in Caenibacter arsenoxydans, a metalloresistant beta-proteobacterium with an unsequenced genome. Biochimie 2006, 88:595-606.

6. Muller D, Medique C, Koechler S, Barbe V, Barakat M, Talla E, Bonnefoy V, Krin E, Arsene-Ploetze F, Carapito C, et al: A tale of two oxidation states: bacterial colonization of arsenic-rich environments. PLoS genetics 2007, 3(4):e53.

7. Weiss S, Carapito C, Cleiss J, Koechler S, Turlin E, Coppee JY, Heymann M, Kugler V, Stauffert M, Cruveiller $S$, et al: Enhanced structural and functional genome elucidation of the arsenite-oxidizing strain Herminiimonas arsenicoxydans by proteomics data. Biochimie 2009, 91:192-203.

8. Alvarez-Martinez CE, Lourenço RF, Baldini RL, Laub MT, Gomes SL: The ECF sigma factor ST is involved in osmotic and oxidative stress responses in Caulobacter crescentus. Mol Microbiol 2007, 66:1240-1255.

9. Muller D, Lièvremont D, Simeonova DD, Hubert JC, Lett MC: Arsenite oxidase aox genes from a metal-resistant beta-proteobacterium. J Bacteriol 2003, 185:135-141.

10. Santini JM, Hoven Vanden RN: Molybdenum-containing arsenite oxidase of the chemolithoautotrophic arsenite oxidizer NT-26. J Bacteriol 2004, 186:1614-1619.

11. Quéméneur $M$, Heinrich-Salmeron $A$, Muller $D$, Lièvremont $D$, Jauzein $M$, Bertin PN, Garrido F, Joulian C: Diversity surveys and evolutionary relationships of $a 0 \times B$ genes in aerobic arsenite-oxidizing bacteria. $A p p$ Environ Microbiol 2008, 74:4567-4573.

12. Cai $L$, Rensing C, Li X, Wang G: Novel gene clusters involved in arsenite oxidation and resistance in two arsenite oxidizers: Achromobacter sp. SY8 and Pseudomonas sp. TS44. App Microbiol Biotechnol 2009, 83(4):715-25.

13. Clingenpeel SR, D'Imperio S, Oduro H, Druschel GK, McDermott TR: Cloning and in situ expression studies of the Hydrogenobaculum arsenite oxidase genes. App Environ Microbiol 2009, 75:3365-3365.

14. Kashyap DR, Botero LM, Franck WL, Hassett DJ, McDermott TR: Complex regulation of arsenite oxidation in Agrobacterium tumefaciens. J Bacteriol 2006, 188:1081-1088

15. Vallenet D, Labarre L, Rouy Z, Barbe V, Bocs S, Cruveiller S, Lajus A, Pascal G Scarpelli $C$, Médigue $C$ : MaGe: A microbial genome annotation system supported by synteny results. Nucleic Acids Res 2006, 34:53-65.

16. Lett M-C, Paknikar K, Lièvremont D: A simple and rapid method for arsenite and arsenate speciation. Biohydrometallurgy - Fundamentals, Technology and Sustainable Development, Part B Amsterdam: Elsevier ScienceJr VSTCaOG 2001, 541-546, (1348 pp). 
17. Mouncey NJ, Mitchenall LA, Pau RN: Mutational analysis of genes of the mod locus involved in molybdenum transport, homeostasis, and processing in Azotobacter vinelandii. J Bacteriol 1995, 177:5294-5302.

18. Peijnenburg WJGM, Jager T: Monitoring approaches to assess bioaccessibility and bioavailability of metals: Matrix issues. Ecotoxicol Environ Saf 2003, 56:63-77.

19. Soutourina OA, Bertin PN: Regulation cascade of flagellar expression in Gram-negative bacteria. FEMS Microbiol Rev 2003, 27:505-523.

20. Studholme DJ, Dixon R: Domain architectures of $\sigma^{54}$-dependent transcriptional activators. J Bacteriol 2003, 185:1757-1767.

21. Rappas M, Schumacher J, Beuron F, Niwa H, Bordes P, Wigneshweraraj $S$, Keetch CA, Robinson CV, Buck M, Zhang X: Structural insights into the activity of enhancer-binding proteins. Science 2005, 307:1972-1975.

22. Ellis PJ, Conrads T, Hille R, Kuhn P: Crystal structure of the $100 \mathrm{kDa}$ arsenite oxidase from Alcaligenes faecalis in two crystal forms at $1.64 \AA$ and 2.03 Å. Structure 2001, 9:125-132.

23. Grunden AM, Shanmugam KT: Molybdate transport and regulation in bacteria. Arch Microbiol 1997, 168:345-354.

24. Parkinson JS, Kofoid EC: Communication modules in bacterial signaling proteins. Annu Rev Genet 1992, 26:71-112.

25. Kustu S, Santero E, Keener J, Popham D, Weiss D: Expression of sigma 54 (ntrA)-dependent genes is probably united by a common mechanism. Microbiol Rev 1989, 53:367-376.

26. Leonhartsberger S, Huber A, Lottspeich F, Böck A: The hydH/G genes from Escherichia coli code for a zinc and lead responsive two-component regulatory system. J Mol Biol 2001, 307:93-105.

27. Barrios $H$, Valderrama $B$, Morett $E$ : Compilation and analysis of $\sigma^{54}$. dependent promoter sequences. Nucleic Acids Res 1999, 27:4305-4313.

28. Schumacher J, Joly N, Rappas M, Zhang X, Buck M: Structures and organisation of AAA+ enhancer binding proteins in transcriptional activation. J Struct Biol 2006, 156:190-199.

29. Zhang X, Chaney M, Wigneshweraraj SR, Schumacher J, Bordes P, Cannon W, Buck M: Mechanochemical ATPases and transcriptional activation. Mol Microbiol 2002, 45:895-903.

30. Yang XF, Alani SM, Norgard MV: The response regulator Rrp2 is essential for the expression of major membrane lipoproteins in Borrelia burgdorferi. Proc Natl Acad Sci Unit States Am 2003, 100:11001-11006.

31. Stafford GP, Scanlan J, McDonald IR, Murell JC: rpoN, mmoR and $m m o G$, genes involved in regulating the expression of soluble methane monooxygenase in Methylosinus trichosporium OB3b. Microbiology 2003, 149:1771-1784.

32. Zhu L, Peng Q, Song F, Jiang Y, Sun C, Zhang J, Huang D: Structure and regulation of the gab gne cluster, involved in the $\gamma$-aminobutyric acid shunt, are controlled by a $\sigma^{54}$ factor in Bacillus thuringiensis. J Bacteriol 2010, 192:346-355

33. Debarbouille M, Gardan R, Arnaud M, Rapoport G: Role of bkdR, a transcriptional activator of the SigL-dependent isoleucine and valine degradation pathway in Bacillus subtilis. J Bacteriol 1999, 181:2059-2066.

34. Dombrecht B, Marchal K, Vanderleyden J, Michiels J: Prediction and overview of the RpoN-regulon in closely related species of the Rhizobiales. Genome Biol 2002, 3(12):RESEARCH0076.

35. Cases I, Ussery DW, De Lorenzo V: The $\sigma^{54}$ regulon (sigmulon) of Pseudomonas putida. Environ Microbiol 2003, 5:1281-1293.

36. Endoh $T$, Habe $H$, Yoshida $T$, Nojiri $H$, Omori T: A CysB-regulated and $\sigma^{54}$ dependent regulator, $\mathrm{SfnR}$, is essential for dimethyl sulfone metabolism of Pseudomonas putida strain DS1. Microbiology 2003, 149:991-1000.

37. Grigoroudis Al, Panagiotidis CA, Lioliou EE, Vlassi M, Kyriakidis DA: Molecular modeling and functional analysis of the AtoS-AtoC twocomponent signal transduction system of Escherichia coli . Biochim Biophys Acta Gen Subj 2007, 1770:1248-1258.

38. Bordes P, Wigneshweraraj SR, Schumacher J, Zhang X, Chaney M, Buck M: The ATP hydrolyzing transcription activator phage shock protein $F$ of Escherichia coli : Identifying a surface that binds $\sigma^{54}$. Proc Natl Acad Sci Unit States Am 2003, 100:2278-2283.

39. Dago AE, Wigneshweraraj SR, Buck M, Morett E: A role for the conserved GAFTGA motif of AAA+ transcription activators in sensing promoter DNA conformation. J Biol Chem 2007, 282:1087-1097.

40. Hartl FU: Molecular chaperones in cellular protein folding. Nature 1996, 381:571-580

41. Mayer MP, Bukau B: Hsp70 chaperone systems: Diversity of cellular functions and mechanism of action. Biol Chem 1998, 379:261-268.
42. Yoon H, Hong J, Ryu S: Effects of chaperones on mRNA stability and gene expression in Escherichia coli. J Microbiol Biotechnol 2008, 18:228-233.

43. Ohki R, Kawamata T, Katoh Y, Hosoda F, Ohki M: Escherichia coli dna」 deletion mutation results in loss of stability of a positive regulator, CRP. J Biol Chem 1992, 267:13180-13184.

44. Berks BC, Sargent F, Palmer T: The Tat protein export pathway. Mol Microbiol 2000, 35:260-274

45. Pérez-Rodriguez R, Fisher AC, Perlmutter JD, Hicks MG, Chanal A, Santini CL, Wu LF, Palmer T, DeLisa MP: An Essential Role for the DnaK Molecular Chaperone in Stabilizing Over-expressed Substrate Proteins of the Bacterial Twin-arginine Translocation Pathway. J Mol Biol 2007, 367:715-730.

46. Rodriguez F, Arsène-Ploetze F, Rist W, Rüdiger S, Schneider-Mergener J, Mayer MP, Bukau B: Molecular Basis for Regulation of the Heat Shock Transcription Factor sigma32 by the DnaK and DnaJ Chaperones. Mol Cell 2008, 32:347-358.

47. De Lorenzo V, Herrero M, Jakubzik U, Timmis KN: Mini-Tn5 transposon derivatives for insertion mutagenesis, promoter probing, and chromosomal insertion of cloned DNA in gram-negative eubacteria. $J$ Bacteriol 1990, 172:6568-6572.

48. Sambrook J, Fritsch EF, Maniatis T: Molecular cloning: a laboratory manual. Cold Spring Harbor Laboratory Press, Cold Spring Harbor, NY, 21989.

49. Smyth GK, Speed TP: Normalization of cDNA microarray data. Methods 2003, 31:265-273.

50. Delmar P, Robin S, Daudin JJ: VarMixt : Efficient variance modeling for the differential analysis of replicated gene expression data. Bioinformatics 2004, 21(4):502-8.

51. Benjamini Y, Yekutieli D: The control of the false discovery rate in multiple hypothesis testing under dependency. Ann Stat 2001, 29:1165-1188.

52. Pfaffl MW: A new mathematical model for relative quantification in realtime RT-PCR. Nucleic Acids Res 2001, 29(9):e45.

53. Pfaffl MW, Horgan GW, Dempfle L: Relative expression software tool (REST) for group-wise comparison and statistical analysis of relative expression results in real-time PCR. Nucleic Acids Res 2002, 30(9):e36.

54. Anderson GL, Williams J, Hille R: The purification and characterization of arsenite oxydase from Alcaligenes faecalis, a molybdenum-containing hydroxylase. J Biol Chem 1992, 267:23674-23682.

doi:10.1186/1471-2180-10-53

Cite this article as: Koechler et al: Multiple controls affect arsenite oxidase gene expression in Herminiimonas arsenicoxydans. BMC Microbiology 2010 10:53.

\section{Submit your next manuscript to BioMed Central and take full advantage of:}

- Convenient online submission

- Thorough peer review

- No space constraints or color figure charges

- Immediate publication on acceptance

- Inclusion in PubMed, CAS, Scopus and Google Scholar

- Research which is freely available for redistribution
Biomed Central 